\title{
ON THE LIMIT OF BLOCK TOEPLITZ DETERMINANTS
}

\section{HAROLD WIDOM ${ }^{1}$}

ABSTRACT. An asymptotic formula is derived for block Toeplitz determinants which generalizes Szegö's well-known formula for ordinary Toeplitz determinants.

The predecessor of this paper [5] was concerned, among other things, with the limiting behavior of the block Toeplitz determinants $D_{N}[\phi]=$ $\operatorname{det} T_{N}[\phi]$ where $T_{N}[\phi]=\left(\phi_{i-j}\right), i, j=0, \cdots, N$. Here

$$
\phi(z)=\sum_{k=-\infty}^{\infty} \phi_{k} z^{k}, \quad|z|=1,
$$

is an $r \times r$ matrix-valued function satisfying

$$
\|\phi\|=\sum_{k=-\infty}^{\infty}\left\|\phi_{k}\right\|+\left\{\sum_{k=-\infty}^{\infty}|k|\left\|\phi_{k}\right\|^{2}\right\}^{1 / 2}<\infty .
$$

The norms appearing on the right side are Hilbert-Schmidt norms of the $r \times r$ matrices which occur.

The set of such functions is a Banach algebra, denoted by $A$. It has a distinguished subset $A_{0}$ consisting of those functions satisfying the conditions

$$
\operatorname{det} \phi\left(e^{i \theta}\right) \neq 0, \quad 0 \leq \hat{\theta} \leq 2 \pi \quad \arg \operatorname{det} \phi\left(e^{i \theta}\right)=0 .
$$

For $\phi \in A$ these conditions are necessary and sufficient that the corresponding semi-infinite Toeplitz matrix

$$
T[\phi]=\left(\phi_{i-j}\right), \quad 0 \leq i, j<\infty,
$$

considered as an operator (acting in the left) on the Hilbert space $l_{2}^{+}$of square-summable semi-infinite sequences of $r$-vectors, is a Fredholm operator of index zero.

Received by the editors December 26, 1973 and, in revised form, April 8, 1974. AMS (MOS) subject classifications (1970). Primary 47B35.

1 Research supported by a grant from the National Science Foundation. 
It was shown in [5] that if one defines

$$
G[\phi]=\exp \left\{\frac{1}{2 \pi} \int_{0}^{2 \pi} \log \operatorname{det} \phi\left(e^{i \theta}\right) d \theta\right\},
$$

then for any $\phi \in A_{0}$ the limit

$$
E[\phi]=\lim _{N \rightarrow \infty} D_{N}[\phi] / G[\phi]^{N+1}
$$

exists. Various properties of the function $E[\phi]$ were derived and expressions were found for it in certain instances. However a general formula, containing the well-known Szegö formula

$$
E[\phi]=\exp \left\{\sum_{k=1}^{\infty} k(\log \phi)_{k}(\log \phi)_{-k}\right\}
$$

in the scalar case $r=1$, was lacking.

In this paper such a formula will be derived. It reads, very simply,

$$
E[\phi]=\operatorname{det} T[\phi] T\left[\phi^{-1}\right] \text {. }
$$

Recall that there is a determinant theory for operators differing from the identity by a nuclear (trace class) operator. It is to this that "det" refers here. (All the facts we shall need about these determinants may be found in [1].)

To see why (1) makes sense, and why it is equivalent to Szegö's formula in the scalar case, consider any $\phi$ and $\psi$ belonging to $A$ and observe that the $i, j$ entry of the semi-infinite matrix $T[\phi \psi]-T[\phi] T[\psi]$ equals

$$
\sum_{k=-\infty}^{\infty} \phi_{i-k} \psi_{k-j}-\sum_{k=0}^{\infty} \phi_{i-k} \psi_{k-j}
$$

Therefore

$$
T[\phi \psi]-T[\phi] T[\psi]=\left(\sum_{k=1}^{\infty} \phi_{i+k} \psi_{-k-j}\right)_{i, j \geq 0} .
$$

This is the product of two semi-infinite matrices each of which is HilbertSchmidt since

$$
\sum_{i=0}^{\infty} \sum_{k=1}^{\infty}\left\|\phi_{i+k}\right\|^{2}=\sum_{k=1}^{\infty} k\left\|\phi_{k}\right\|^{2}<\infty
$$

and similarly for the matrix arising from $\psi$. Thus the operator $T[\phi \psi]-$ $T[\phi] T[\psi]$ is always nuclear. Taking $\psi=\phi^{-1}$ one concludes that $T[\phi] T\left[\phi^{-1}\right]$ differs from $I$ by a nuclear operator and so its determinant is defined. 
Write

$A^{+}=\left\{\phi \in A: \phi_{k}=0\right.$ for $\left.k<0\right\}, A^{-}=\left\{\phi \in A: \phi_{k}=0\right.$ for $\left.k>0\right\}$.

In case either $\phi \in A^{-}$or $\psi \in A^{+}$the right side of (2) vanishes so

$$
T[\phi \psi]=T[\phi] T[\psi] \text { if } \phi \in A^{-} \text {or } \psi \in A^{+} .
$$

In particular the mapping

$$
\phi \rightarrow T[\phi]
$$

is an isomorphism from either of the subalgebras $A^{ \pm}$into the algebra of operators on $l_{2}^{+}$.

Suppose now that there is a factorization $\phi=e^{\psi-} e^{\psi^{+}}$where $\psi^{ \pm} \in A^{ \pm}$ and $\psi^{-}$and $\psi^{+}$commute. Then (3) and (4) give

$$
\begin{aligned}
T[\phi] T\left[\phi^{-1}\right] & =T\left[e^{\psi^{-}}\right] T\left[e^{\psi^{+}}\right] T\left[e^{-\psi^{-}}\right] T\left[e^{-\psi^{+}}\right] \\
& =e^{T\left[\psi^{-}\right]} e^{T\left[\psi^{+}\right]} e^{-T\left[\psi^{-}\right]} e^{-T\left[\psi^{+}\right]} .
\end{aligned}
$$

Thus $T[\phi] T\left[\phi^{-1}\right]$ is a multiplicative commutator of exponentials of Toeplitz operators.

Now there is an elegant formula which expresses the determinant of the multiplicative commutator of the exponentials of two operators in terms of the trace of their additive commutator in case the latter is nuclear:

$$
\operatorname{det} e^{W} e^{Q} e^{-W} e^{-Q}=\exp \operatorname{tr}(W Q-Q W) .
$$

(The formula appears in [3] and $[2, \$ 10]$. The latter paper hints at a possible connection with Szegö's formula and this in fact inspired the present work.). In our case $W=T\left[\psi^{-}\right], Q=T\left[\psi^{+}\right]$. The formula is applicable, since by (3) and the commutativity of $\psi^{-}$and $\psi^{+}$,

$$
T\left[\psi^{-}\right] T\left[\psi^{+}\right]-T\left[\psi^{+}\right] T\left[\psi^{-}\right]=T\left[\psi^{+} \psi^{-}\right]-T\left[\psi^{+}\right] T\left[\psi^{-}\right]
$$

which we know is nuclear. Combining this with (5) and (6) and using (2) give immediately

$$
\operatorname{det} T[\phi] T\left[\phi^{-1}\right]=\exp \left\{\operatorname{tr} \sum_{k=1}^{\infty} k\left(\psi^{+}\right)_{k}\left(\psi^{-}\right)_{-k}\right\} .
$$

Thus (1) is equivalent to Szegö's formula in the scalar case where the required factorization always exists, and gives an analogue of it in some instances of the matrix case. 
Once the formula (1) is guessed its proof follows without much difficulty from the results of [5]. Define

$$
A_{0}^{+}=A_{0} \cap A^{+}, \quad A_{0}^{-}=A_{0} \cap A^{-}
$$

(these are the sets of invertible elements of the subalgebras $A^{ \pm}$) and write $A_{1}$ for the subset of $A_{0}$ consisting of those $\phi$ for which there are factorizations

$$
\phi^{-1}=u^{+} u^{-}=v^{-} v^{+}
$$

with $u^{+}, v^{+} \in A_{0}^{+}, u^{-}, v^{-} \in A_{0}^{-}$. Equivalently $\phi \in A_{1}$ if and only if both $T[\phi]$ and $T\left[\phi^{-1}\right]$ are invertible $[5, \S 2]$. Thus $A_{1}$ is an open subset of $A_{0}$. It is also dense in $A_{0}$ [5, Lemma 2.3] and connected [5, Lemma 2.2]. Since both sides of (1) are continuous functions on $A_{0}$ [5, Theorem 4.2], $\left[1\right.$, p. 160], we need only consider $\phi$ belonging to $A_{1}$.

Theorem 4.1 of $[5]$ says that if $\phi \in A_{1}$ then $E[\phi] \neq 0$ and, moreover, if $t \rightarrow \phi(t)$ is a differentiable function from a real interval to $A_{1}$ then $\log E[\phi(t)]$ is a differentiable function of $t$ with derivative equal to

$$
-\operatorname{tr} \sum_{i=-\infty}^{\infty} \phi_{i}^{\prime} \sum_{m=1}^{\infty} m u_{m}^{+} u_{-i-m}^{-}-\operatorname{tr} \sum_{i=-\infty}^{\infty} \phi_{-i}^{\prime} \sum_{m=1}^{\infty} m v_{-m}^{-} v_{i+m}^{+} .
$$

(The dependence on $t$ of the last expression is not displayed. The prime denotes differentiation with respect to $t$ and the functions $u^{ \pm}, v^{ \pm}$refer to the factorization (8) with $\phi=\phi(t)$.)

Formula (2) shows that for any $\phi, \psi \in A$,

$$
\operatorname{tr}(T[\phi \psi]-T[\phi] T[\psi])=\operatorname{tr} \sum_{m=1}^{\infty} m \phi_{m} \psi_{-m}
$$

from which it follows easily that (9) is equal to

$$
\operatorname{tr}\left(T\left[u^{+}\right] T\left[u^{-} \phi^{\prime}\right]-T\left[u^{+} u^{-} \phi^{\prime}\right]\right)+\operatorname{tr}\left(T\left[v^{+} \phi^{\prime}\right] T\left[v^{-}\right]-T\left[v^{+} \phi^{\prime} v^{-}\right]\right) .
$$

Use of (3) and (4) shows that the first term of (10) is equal to the trace of

$$
\begin{aligned}
T\left[u^{+}\right] T\left[u^{-}\right] T\left[\phi^{\prime}\right]-T\left[\phi^{-1} \phi^{\prime}\right] & =T\left[\left(u^{+} u^{-}\right)^{-1}\right]^{-1} T\left[\phi^{\prime}\right]-T\left[\phi^{-1} \phi^{\prime}\right] \\
& =T[\phi]^{-1} T\left[\phi^{\prime}\right]-T\left[\phi^{-1} \phi^{\prime}\right] .
\end{aligned}
$$

Since the trace is a similarity invariant [1, p. 99] the second term of (10) is equal to the trace of 


$$
\begin{aligned}
T\left[v^{-}\right] T\left[v^{+} \phi^{\prime}\right]-T\left[v^{-}\right] & T\left[v^{+} \phi^{\prime} v^{-}\right] T\left[v^{-}\right]^{-1} \\
& =T\left[v^{-} v^{+} \phi^{\prime}\right]-T\left[v^{-} v^{+} \phi^{\prime} v^{-} v^{+}\right] T\left[v^{+}\right]^{-1} T\left[v^{-}\right]^{-1} \\
& =T\left[\phi^{-1} \phi^{\prime}\right]-T\left[\phi^{-1} \phi^{\prime} \phi^{-1}\right] T\left[\phi^{-1}\right]^{-1} .
\end{aligned}
$$

Thus $(d / d t) \log E[\phi(t)]$, which is given by (9), is equal to

$$
\operatorname{tr}\left(T[\phi]^{-1} T\left[\phi^{\prime}\right]-T\left[\phi^{-1} \phi^{\prime} \phi^{-1}\right] T\left[\phi^{-1}\right]^{-1}\right) .
$$

The next step, which is routine, is the verification that

$$
d\left(\log \operatorname{det} T[\phi] T\left[\phi^{-1}\right]\right) / d t
$$

is given by the same expression. We use the general fact that for any differentiable function $S(t)$ from a real interval into the set of nuclear operators one has

$$
\frac{d}{d t} \log \operatorname{det}[I-S(t)]=-\operatorname{tr}[I-S(t)]^{-1} S^{\prime}(t) .
$$

(See [1, p. 163] where this is proved for holomorphic functions, although the formula holds in full generality. Actually, as will be seen, only holomorphic functions need be used.) In our case

$$
S(t)=I-T[\phi(t)] T\left[\phi(t)^{-1}\right]
$$

and we find that

$$
\begin{aligned}
& \frac{d}{d t} \log \operatorname{det} T[\phi] T\left[\phi^{-1}\right] \\
& \quad=\operatorname{tr} T\left[\phi^{-1}\right]^{-1} T[\phi]^{-1}\left(T\left[\phi^{\prime}\right] T\left[\phi^{-1}\right]-T[\phi] T\left[\phi^{-1} \phi^{\prime} \phi^{-1}\right]\right) .
\end{aligned}
$$

Another application of the invariance of the trace under similarity shows that this equals

$$
\begin{aligned}
\operatorname{tr} T[\phi]^{-1}\left(T\left[\phi^{\prime}\right]\right. & \left.T\left[\phi^{-1}\right]-T[\phi] T\left[\phi^{-1} \phi^{\prime} \phi^{-1}\right]\right) T\left[\phi^{-1}\right]^{-1} \\
& =\operatorname{tr}\left(T[\phi]^{-1} T\left[\phi^{\prime}\right]-T\left[\phi^{-1} \phi^{\prime} \phi^{-1}\right] T\left[\phi^{-1}\right]^{-1}\right)
\end{aligned}
$$

as desired.

Thus

$$
\frac{d}{d t}\left(\log E[\phi]-\log \operatorname{det} T[\phi] T\left[\phi^{-1}\right]\right)=0
$$

for any differentiable function $\phi(t)$ with range in $A_{1}$. In particular $\log E[\phi]$ - log det $T[\phi] T\left[\phi^{-1}\right]$ is constant on any line segment in $A_{1}$. Since $A_{1}$ is an open connected subset of $A$ this difference is constant on all of $A_{1}$, and 
the constant must be zero since the difference is trivially zero if $\phi$ is the identity of $A$. This completes the proof of (1).

Finally we mention another situation where a formula similar to (7) holds. Suppose that $\phi$ has a factorization

$$
\phi=\phi^{-} \phi^{+}, \quad \phi^{-} \in A_{0}^{-}, \phi^{+} \in A_{0}^{+},
$$

where each coefficient of $\phi^{-}$commutes with each coefficient of $\phi^{+}$. It need not be true that $\phi^{ \pm}$have logorithms in $A^{ \pm}$. (There exist analytic invertible matrix-valued functions in the unit disc without analytic logarithms.) Nevertheless there are substitutes for logarithms that can be used and with these (7) remains valid.

Consideration of the matrix functions

$$
\phi_{t}^{+}(z)=\phi^{+}(t z), \quad \phi_{t}^{-}(z)=\phi^{-}\left(t^{-1} z\right), \quad 0 \leq t \leq 1,
$$

shows that $\phi^{+}$and $\phi^{-}$are homotopic in $A_{0}^{+}$and $A_{0}^{-}$, respectively, to constant invertible matrix functions. It is an exercise in the Jordan canonical form to check that any invertible $r \times r$ matrix is homotopic to the identity through invertible elements of the algebra generated by the original matrix.

In any Banach algebra each element homotopic to the identity through invertible elements is a finite product of exponentials [4, Theorem 1.4.10]. Consequently if $\tilde{A}^{+}$is the subalgebra of $A^{+}$generated by the $\phi_{t}^{+}$, and if $\tilde{A}^{-}$is defined similarly, then there exist factorizations

$$
\phi^{+}=\phi_{1}^{+} \cdots \phi_{m}^{+}, \quad \phi^{-}=\phi_{1}^{-} \cdots \phi_{n}^{-}
$$

where each $\phi_{p}^{+}\left(\right.$resp. $\left.\phi_{q}^{-}\right)$has a logarithm in $\tilde{A}^{+}\left(\right.$resp. $\left.\tilde{A}^{-}\right)$.

The multiplicative identity

$$
\begin{aligned}
& \operatorname{det} T\left[\phi_{1} \phi_{2} \phi_{3}\right] T\left[\left(\phi_{1} \phi_{2} \phi_{3}\right)^{-1}\right] \\
& \quad=\operatorname{det} T\left[\phi_{1} \phi_{2}\right] T\left[\left(\phi_{1} \phi_{2}\right)^{-1}\right] \operatorname{det} T\left[\phi_{2} \phi_{3}\right] T\left[\left(\phi_{2} \phi_{3}\right)^{-1}\right],
\end{aligned}
$$

which holds if $\phi_{1}, \phi_{3} \in A_{0}^{+}, \phi_{2} \in A_{0}^{-}$or $\phi_{1}, \phi_{3} \in A_{0}^{-}, \phi_{2} \in A_{0}^{+}$, readily follows upon application of (3) and the invariance of the determinant under similarity $[1, p .162]$. This identity together with the commutativity of the $\phi_{p}^{+}$ with the $\phi_{q}^{-}$gives

$$
\operatorname{det} T[\phi] T\left[\phi^{-1}\right]=\prod_{p, q} \operatorname{det} T\left[\phi_{p}^{+} \phi_{q}^{-}\right] T\left[\left(\phi_{p}^{+} \phi_{q}^{-}\right)^{-1}\right] \text {. }
$$

Application of the previously derived formula (7) shows that (12) can be written 


$$
\operatorname{det} T[\phi] T\left[\phi^{-1}\right]=\exp \left\{\operatorname{tr} \sum_{k=1}^{\infty} k\left(\psi^{+}\right)_{k}\left(\psi^{-}\right)_{-k}\right\}
$$

if $\psi^{+}$denotes now the sum of the logarithms of the $\phi_{p}^{+}$and $\psi^{-}$the sum of the logarithms of the $\phi_{q}^{-}$.

In case one of the factors $\phi^{-}$or $\phi^{+}$in (11) is a scalar-valued function times the identity matrix the last expression is equal to

$$
\exp \left\{r^{-1} \sum_{k=1}^{\infty} k(\log \operatorname{det} \phi)_{k}(\log \operatorname{det} \phi)_{-k}\right\} \text {. }
$$

This formula for $E[\phi]$ was derived in [5].

\section{REFERENCES}

1. I. C. Gohberg and M. G. Krein, Introduction to the theory of linear nonselfadjoint operators, "Nauka", Moscow, 1965; English transl., Transl. Math. Monographs, vol. 18, Amer. Math. Soc., Providence, R. I., 1969. MR 36 \#3137; 39 \#7447.

2. J. W. Helton and R. E. Howe, Integral operators: traces, index, and homology, Proc. Conf. on Operator Theory, Lecture Notes in Math:, vol. 345, Springer-Verlag, New York, 1973.

3. J. D. Pincus, On the trace of commutators in the algebra of operators generated by an operator with trace class self-commutator, 1972 (unpublished).

4. C. E. Rickart, General theory of Banach algebras, University Series in Higher Math., Van Nostrand, Princeton, N. J。, 1960。 MR 22 \#5903.

5. H. Widom, Asymptotic behavior of block Toeplitz matrices and determinants, Advances in Math. 13 (1974), 284-322.

DEPARTMENT OF MATHEMATICS, UNIVERSITY OF CALIFORNIA AT SANTA CRUZ, SANTA CRUZ, CALIFORNIA 95060 